\title{
Study on the Efficiency and the Cooperative Direction of Tourism and Leisure Industry in Guangdong-Hong Kong-Macao Greater Bay Area
}

\author{
Lanqi Zeng \\ College of Economics, Jinan University, Guangzhou, China \\ Email: zenglanqi@hotmail.com
}

How to cite this paper: Zeng, L.Q. (2018) Study on the Efficiency and the Cooperative Direction of Tourism and Leisure Industry in Guangdong-Hong Kong-Macao Greater Bay Area. Open Journal of Social Sciences, 6, 93-105.

https://doi.org/10.4236/jss.2018.61007

Received: December 11, 2017

Accepted: January 16, 2018

Published: January 19, 2018

Copyright $\odot 2018$ by author and Scientific Research Publishing Inc. This work is licensed under the Creative Commons Attribution International License (CC BY 4.0).

http://creativecommons.org/licenses/by/4.0/ (c) (i) Open Access

\begin{abstract}
The bay area economy is of strategic importance to the progress of the world economy. As an important engine of national reform and opening up, the accelerated development of Guangdong-Hong Kong-Macao Greater Bay Area is an important part of China's national strategy. Drawing on the experience of the economic development in other successful bay area, it is clear that the construction of the Guangdong-Hong Kong-Macao Greater Bay Area should focus on the tourism and leisure industry. Based on the government statistics, a gray relational model and a vector auto-regression model (VAR) are employed to analyze the efficiency of the Tourism and Leisure Industry in Guangdong-Hong Kong-Macao Greater Bay Area. The results show that the progress of Tourism and Leisure Industry has a great driving effect on the other related industries, and there exists an interdependence between the tourism and leisure industry, GDP and investment in fixed assets within Guangdong-Hong Kong-Macao Greater Bay Area. It suggests that the government should take appropriate measures to promote cooperation and take corresponding industrial policies to boost the development of the Tourism and Leisure Industry in Guangdong-Hong Kong-Macao Greater Bay.
\end{abstract}

\section{Keywords}

Guangdong-Hong Kong-Macao Greater Bay Area, Tourism and Leisure Industry, Grey Correlation, VAR

\section{Introduction}

As the dynamic expanding of the global economy, national governments and 
academic circles show great attention to the exploitation of Bay Area. The word "Bay Area" stems from San Francisco Bay Area. Nowadays, the world has basically formed eight well-known Bay Area, New York Bay Area, San Francisco Bay Area and Tokyo Bay Area are the most matured and influential Bay Area among them. For instance, New York Bay Area, hub of the mainstay of the American economy, known as the nerve center of the international finance. Its urbanization level has remained above $90 \%$ and manufacturing output accounts for $30 \%$ of the national output [1]. Today, Bay Area economy has become a strategic platform for world's economic development. However, compared with overseas, the construction of Bay Area in China is started relatively late.

The "Hong Kong Bay Area" proposed by Wu Jiawei in the 1980s is considered to be the earliest idea of building a Bay Area in China. The formal appearance of "Bay Area" is the "Coordinated Development Planning of Pearl River Delta Township Group (2004-2020)" promulgated in 2005. For the first time, "Greater Bay Area" was explicitly put forward in 2015 by the National Development and Reform Commission, the Ministry of Foreign Affairs and the Ministry of Commerce in "Vision and Action on Jointly Building Silk Road Economic Belt and 21st-Century Maritime Silk Road". March 2016, the State Council issued "Guiding opinions on deepening regional cooperation in the Pan Pearl River Delta", the Regional Cooperation in Pan-Pearl River Delta has since become a national strategy. "Guiding opinions" explicitly required that brought into full play the radiating exemplary role of Guangzhou and Shenzhen, Guangdong is working in association with Hong Kong and Macao to build Guangdong-Hong Kong-Macao Greater Bay Area and World-class City Groups, Structuring significant economic support belts which based on Guangdong-Hong Kong-Macao Greater Bay Area and backed up by the Zhu-jiang and $\mathrm{Xi}$-jiang economic belt, stimulating the development of the southwest and south-central China, radiating Southeast Asia and South Asia. In 2017, Premier Li Keqiang proposed that it is necessary to study and formulate a strategic planning on the development of urban agglomeration in Guangdong-Hong Kong-Macao Greater Bay Area. Thus, it can be seen that our government attached great importance to the development of Guangdong-Hong Kong-Macao Greater Bay Area. What's more, regarding as the forward position of reforming and opening-up, Guangdong-Hong Kong-Macao Greater Bay Area becomes an important engine for economic progress. Learning from international experience and combining the actual condition, the construction of our Bay Area should highlight the tourism and leisure industry.

At present, the scholar's general ideas about the economic impact of the tourism and leisure industry are as follows: Li Jiangfan made a quantitative analysis related to tourism industry based on the Input-Output Table of Guangdong, considered that tourism industry have strong industrial relevance, consumption complementarity and industrial effect [2]. Song Zengwen held that China's tourism industry had a relatively strong forward driving effect [3]. Weng Gangmin 
analyzed the particular character of the industry association of the tourism industry, the results show that it has low intermediate demand but high input rate, weak prior correlation but strong backward correlation [4]. Wu Sanmang thought that the tourism industry is closely related to other industries of the national economy and the proportion of related industries is on the rise [5]. Wang Qiyan analyzed the correlation between tourism and agriculture in Beijing, it showed that the level of agricultural development had a certain influence on tourism, but the correlation degree was relatively low [6]. According to the existing literature, the paper about tourism and leisure industrial function in Guangdong-Hong Kong-Macao Greater Bay Area is rarely found up to now. Therefore, this paper analyzes the driving effect of tourism and leisure industry in Guangdong-Hong Kong-Macao Greater Bay Area.

In the perspective of geography, "Bay Area" is a particular urban space of coastal city or urban agglomeration. It is used to describe the port group and urban agglomeration formed by many seaports and towns distributed around coastal ports [7]. Based on the characteristics of Bay Area, this paper's research scope includes Hong Kong, Macao, Guangzhou, Shenzhen, Zhuhai, Foshan, Dongguan, Zhongshan, Zhaoqing, Huizhou and Jiangmen, for a total of 9 cities and 2 Special Administrative Regions. Obviously, the benefits that can be obtained through strengthening the research on tourism and leisure industry in Guangdong-Hong Kong-Macao Greater Bay Area and grasping its regularity and orientation of construction should not be underestimated.

At present, most of the studies on Guangdong-Hong Kong-Macao Greater Bay Area are qualitative analysis-based, which is lack of quantitative analysis on the tourism and leisure industry in this area. Thus, it is difficult to identify the importance that the tourism and leisure industry played at a deeper level. Based on this, it is of practical significance that the paper analyzes the driving efforts of the tourism and leisure industry in Guangdong-Hong Kong-Macao Greater Bay Area quantitatively.

\section{Theoretical Basis}

\subsection{Tourism and Leisure Industry Is an Inevitable Requirement}

The Bay Area consists of a single bay or a number of coves, harbors and adjacent islands. Nowadays, the world's most developed regions tend to be concentrated around the Bay Area, such as New York Bay Area, San Francisco Bay Area and Tokyo Bay Area et cetera. Tourism and leisure industry is an inevitable requirement for the construction of the Guangdong-Hong Kong-Macao Greater Bay Area. The observation of the industrial structure changes of the international Bay area shows that coastal tourism industry has become one of the largest and fastest growing industry in the world: 1) The proportion of the added value of the primary, secondary and tertiary industries in New York Bay Area is 0:10.65:89.35 (in the year of 2013), and Coastal tourism is an important part of the tertiary industry; 2) As for the Busan Bay Area, it emphasizes the develop- 
ment of coastal industry in the latest marine economic development strategy; 3) In Singapore, the coastal industry has become one of the three leading industries, surpassed the output of Marine secondary industry, which covered Marine vessel manufacturing, maintenance and export-oriented industries. What's more, tourism has now become one of the major sources of foreign exchange in the Gulf of Singapore. The development of tourism has also led to the development of other industries and increased employment opportunities; 4) The output value of Coastal tourism in the United States San Francisco Bay Area accounted for over four fifths of the total marine economic output value.

\subsection{Tourism and Leisure Industry Is an Objective Requirement of Building an Ocean Strong Province in Guangdong}

Guangdong Province, face the South China Sea, direct to the Pacific Ocean, equipped with the international maritime thoroughfare which envied by other coastal provinces makes it the first-class marine province. Accelerate the development of marine economy is the feature, advantage and potential of Guangdong. Take Zhuhai for example, according to its statistics of marine economy, the total output value of marine industry reached 70.46 billion yuan in 2013, among them, the coastal tourism reached 24.18 billion yuan, accounting for $34 \%$ of the total output value. Compared with the national situation, the Coastal tourism revenue in the Mainland reached 888.2 billion yuan in 2014, accounting for $35.3 \%$ of the total output value of the marine industry. However, that proportion of San Francisco Bay Area is 85.3\%, which show the extreme importance of speed up the process of building Guangdong a strong ocean province and focus on the coastal tourism.

\subsection{Tourism and Leisure Industry Contribute to Build the World's Tourism and Leisure Center in Hong Kong and Macao}

Hong Kong occupy an important position in the world's tourism and leisure center and has become an internationally important tourist and leisure destination with its comprehensive shipping and transportation system, excellent catering and hotel services, unique culture, loose and appropriate immigration policies, strong economic strength and strong tourism development awareness. Since Hong Kong's return to the motherland in 1997, the implementation of the special government policy of "one country, two systems" and especially the "free exercise" has brought new impetus and new opportunities to the development of Hong Kong's international leisure and tourism hub. The construction of World Tourism and Leisure Center in Macao is a national strategy. In 2008, the "Plan for Reform and Development of the Pearl River Delta Region" promulgated by the National Development and Reform Commission first identified Macao as a world tourism and leisure center. In November 2010, Wen Jiabao, then Premier of the State Council, proposed "supporting the establishment of the Macao World Tourism and Leisure Center". In 2011, the "12th Five-Year Plan" and the 
"Framework Agreement for Cooperation between Guangdong and Macao" made clear that "support the establishment of a world tourism and leisure center in Macao and promote the development of a moderately diversified economy". All of these policies integrate the international leisure tourism center of the Macao into the national development strategy. The construction of Guangdong-Hong Kong-Macao Greater Bay Area will highlight and promote the status of Hong Kong and Macao in developing the World Tourism and Leisure Center.

\section{Analysis of Current Situation}

As far as the tourism industry is concerned, the tourism resources of the three places complement each other with a solid foundation of tourism cooperation and a good relationship. In 2016, the number of tourist arrivals in Guangdong, Hong Kong, Macao reached 323.6913 million, total tourism revenue increased from 265.847 billion yuan in 2005 to 976.612 billion yuan in 2016 (Figure 1) with an average annual growth rate of $12.56 \%$, reached a remarkable achievement. Nowadays, the state attaches great importance to and supports the development of the Guangdong-Hong Kong-Macao Greater Bay Area, and brings unprecedented opportunities to the development of the tourism and leisure industry.

Guangdong-Hong Kong-Macao Greater Bay Area including the following 11 cities: Hong Kong city, Macao city, Guangzhou city, Shenzhen city, Zhuhai city, Foshan city, Dongguan city, Zhongshan city, Zhaoqing city, Huizhou city and Jiangmen city. In 2016, Guangzhou city ranked first among those 11 cities in terms of both tourism revenue (321.7 billion yuan) and tourist receipts (60 million). Hong Kong city's tourism revenue grew rapidly from 79.814 billion yuan in 2005 to 263.932 billion yuan in 2014, but declined slightly in recent two years. In 2016, its tourism revenue was 204.8 billion yuan, ranked second. As for Shenzhen city, its tourism revenue continues to grow, received 136.866 billion in

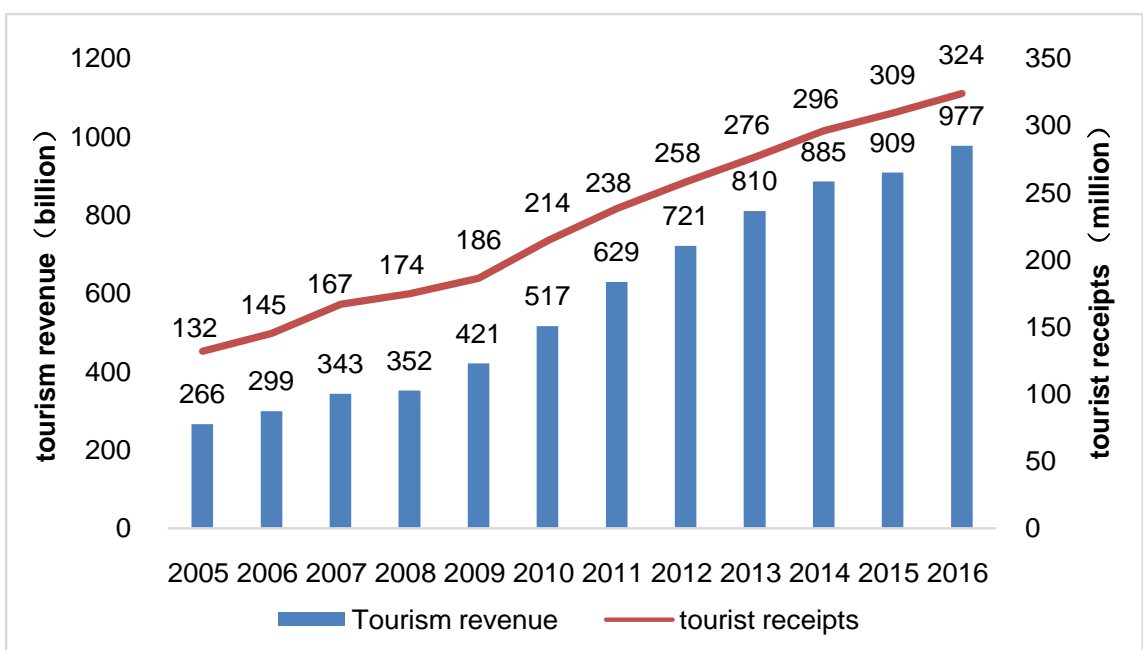

Figure 1. Tourism revenue and tourist receipts in Guangdong-Hong Kong-Macao greater bay area. 
2016, accounting for $14 \%$ of the overall revenue of Guangdong-Hong Kong-Macao Greater Bay Area, ranked third. Macao city's tourism revenue has undergone an undulated growth, obtained 43.8 billion yuan. Followed by Foshan city, which revenue achieved 62.47 billion yuan and received 13.51 million tourists. Although Huizhou city and Zhaoqing city acquired less revenue, their annual growth rate run up to $19.79 \%$ and 19.24 respectively, come out in front of the other cities. It is noteworthy that the tourist reception volume of Zhaoqing City plummeted in 2014, but gradually picked up in 2016, showing strong momentum of growth simultaneously.

In the past five years, the gross domestic product of Guangdong-Hong Kong-Macao Greater Bay Area continued to grow. In the course of rapid economic development, a large number of middle- and high-income earners were cultivated. Higher disposable income, leisure and beautiful coastal scenery, these three key factors for the development of cruise tourism and yacht tourism have been formed. Therefore, apart from the traditional coastal tourism projects such as coast co-travel and island vacation tourism, cruise tourism and yacht tourism, which have strong pulling power and adsorption capacity, have become the economic growth points for the tourism and leisure industry in Guangdong-Hong Kong-Macao Greater Bay Area.

Cruise tourism, connect with both the manufacturing industry and the modern service industry, possessed huge industrial chain and has huge potential international tourism market. Currently, Guangzhou city has opened cruise lines to ASEAN countries. In 2016, it operated a total of 103 cruise ships and received 323,600 tourists, jumped to third in the country within a year. The main cruise home port project in Nansha District plans to build a 225,000-ton cruise berth and a 100,000-ton cruise berth. Upon completion, it will become one of the largest cruise home ports in China. In addition, the prince's Bay Cruise Terminal in Shenzhen has been completed and has the world's largest cruise terminal capable of calling for a total of 220,000-tonne vessels and was approved to set up the "China Cruise Tourism Development Experimental Zone".

In the Guangdong-Hong Kong-Macao Greater Bay Area, the output value of Yacht manufacturing in Zhuhai city, Zhongshan city and Jiangmen city account for half of the country. Hong Kong city, own more than a hundred years of yacht culture; Shenzhen city, occupied the largest yacht consumer market; Pearl River Estuary and Daya Bay area, possessed Yacht port consumption format. Combining all the strength above, formed a complete chain from manufacturing, service to consumer terminals, laid a good foundation for the development of the yacht industry. November 23, 2016, the free yacht between Zhongshan city and Macao city was formally opened to navigation, Zhongshan became the first mainland city to open a free exercise of yachts with the Macao SAR. After that, Zhongshan city will also promote the successful experience of expanding the free exercise of the yachts with Macao and gradually extend the scope of free exercise to Hong Kong and neighboring countries. The yacht industry will become a po- 
werful engine for accelerating Zhongshan's industrial upgrading and helping Macao to achieve a moderate diversified development. At present, the infrastructure of yachts in the Guangdong-Hong Kong-Macao Greater Bay Area is becoming more and more sophisticated. Moreover, Shenzhen and other cities have enrolled the construction of Public yacht wharf into Tourism infrastructure development plan.

In addition, some tourism projects with large-scale and high-quality will promote the development of the tourism and leisure industry and have greatly pushed forward the upgrading and advancement of Tourism image in Guangdong-Hong Kong-Macao Greater Bay Area. The modern, and large-scale theme park has become an important tourism card, and the gradual extension of culture, hotel, film and television, real estate and other industrial chains make these theme parks become the symbol of the city's hard and soft power. Of the top 10 global theme parks operated in 2015, three were headquartered in the Guangdong-Hong Kong-Macao Greater Bay Area, among them, as the forerunners of the development and innovation of domestic theme parks, Overseas Chinese Town in Shenzhen received 30.18 million tourists, ranked fourth in the world. Chimelong Group ranked seventh with 23,587,000 tourist arrivals, followed by Shenzhen Huaqiang Square.

Overall, the economy of Guangdong-Hong Kong-Macao Greater Bay Area develops rapidly, and tourism and leisure industry showing strong growth too. Among them, the coastal tourism projects, especially cruise tourism, yacht tourism and theme park tourism will give renewed impetus to the expanding of tourism and leisure industry in Guangdong-Hong Kong-Macao Greater Bay Area.

\section{Empirical Analysis}

\subsection{Data Sources}

This paper selects data from 2005 to 2016 to analyze the driving effect of the tourism and leisure industry in Guangdong-Hong Kong-Macao Greater Bay Area. The data in this paper come from China Statistical Yearbook, Guangzhou Statistical Yearbook, Shenzhen Statistical Yearbook, Zhuhai Statistical Yearbook, Dongguan Statistical Yearbook, Zhongshan Statistical Yearbook, Huizhou Statistical Yearbook, Jiangmen Statistical Yearbook, Foshan Statistical Yearbook, Zhaoqing Statistical Yearbook, Hong Kong Statistical Yearbook, Macao Statistical Yearbook and the websites of municipal statistical offices and the statistical bulletins of national economic and social development of all cities.

\subsection{Grey Correlation Analysis}

At first, this paper analyzes the industrial driving force of the tourism and leisure industry in Guangdong-Hong Kong-Macao Greater Bay Area. Gray correlation degree is a quantitative description of the correlation between the various factors, the basic idea is to calculate the degree of correlation according to the 
closeness of factors varies with time. Calculation methods are as follows:

Original sequence:

$$
X_{0}=\left(x_{0}(1), x_{0}(2), \cdots, x_{0}(n)\right) \text { and } X_{1}=\left(x_{1}(1), x_{1}(2), \cdots, x_{1}(n)\right) \text {, }
$$

make start point zero, turn it to be $\boldsymbol{X}_{\mathbf{0}}^{\prime}=\left(\boldsymbol{X}_{\mathbf{0}}^{\prime}(1), \boldsymbol{X}_{\mathbf{0}}^{\prime}(2), \cdots, \boldsymbol{X}_{\mathbf{0}}^{\prime}(n)\right)$ and $\boldsymbol{X}_{\mathbf{1}}^{\prime}=\left(\boldsymbol{X}_{\mathbf{1}}^{\prime}(1), \boldsymbol{X}_{\mathbf{1}}^{\prime}(2), \cdots, \boldsymbol{X}_{\mathbf{1}}^{\prime}(n)\right)$, obtain $S_{0}$ and $S_{1}$ :

$$
\begin{gathered}
S_{0}=\frac{1}{2} \sum_{k=2}^{n}\left[x_{0}^{\prime}\left(n_{k-1}\right)+x_{0}^{\prime}\left(n_{k}\right)\right] \\
S_{1}=\frac{1}{2} \sum_{k=2}^{n}\left[x_{1}^{\prime}\left(n_{k-1}\right)+x_{1}^{\prime}\left(n_{k}\right)\right]
\end{gathered}
$$

Calculate absolute and relative degree $\boldsymbol{e}_{\mathbf{0 1}}$ and $\boldsymbol{e}_{\mathbf{0 1}}^{\prime}$ :

$$
\begin{aligned}
& e_{01}=\frac{\left(1+\left|S_{0}\right|+\left|S_{1}\right|\right)}{\left(1+\left|S_{0}\right|+\left|S_{1}\right|+\left|S_{0}-S_{1}\right|\right)} \\
& e_{01}^{\prime}=\frac{\left(1+\left|S_{0}^{\prime}\right|+\left|S_{1}^{\prime}\right|\right)}{\left(1+\left|S_{0}^{\prime}\right|+\left|S_{1}^{\prime}\right|+\left|S_{0}^{\prime}-S_{1}^{\prime}\right|\right)}
\end{aligned}
$$

among them $S_{9}^{\prime}=S_{0} / x_{0}(1), \quad S_{1}^{\prime}=S_{1} / x_{1}(1)$, and Calculate synthetic degree:

$$
\boldsymbol{E}_{\mathbf{0 1}}=\frac{e_{01}+e_{01}^{\prime}}{2}
$$

\begin{tabular}{|c|c|c|c|c|}
\hline \multirow[b]{2}{*}{ Region } & \multicolumn{4}{|c|}{ Industry } \\
\hline & Construction & $\begin{array}{c}\text { Wholesale, Retail and } \\
\text { Hotel catering }\end{array}$ & $\begin{array}{l}\text { Transportation and } \\
\text { Telecommunications }\end{array}$ & $\begin{array}{c}\text { Financial, Real estate } \\
\text { and other services }\end{array}$ \\
\hline Greater Bay Area & 0.942 & 0.920 & 0.555 & 0.876 \\
\hline Guangzhou & 0.968 & 0.863 & 0.613 & 0.806 \\
\hline Shenzhen & 0.651 & 0.717 & 0.869 & 0.630 \\
\hline Zhuhai & 0.851 & 0.733 & 0.526 & 0.823 \\
\hline Foshan & 0.925 & 0.821 & 0.691 & 0.768 \\
\hline Dongguan & 0.714 & 0.841 & 0.812 & 0.886 \\
\hline Zhongshan & 0.880 & 0.746 & 0.740 & 0.762 \\
\hline Zhaoqing & 0.701 & 0.826 & 0.554 & 0.837 \\
\hline Jiangmen & 0.671 & 0.730 & 0.605 & 0.819 \\
\hline Huizhou & 0.676 & 0.884 & 0.806 & 0.802 \\
\hline Hong Kong & 0.580 & 0.641 & 0.443 & 0.786 \\
\hline Macao & 0.749 & 0.751 & 0.779 & 0.828 \\
\hline
\end{tabular}

The calculation results of the correlation between tourism and related industries in Guangdong-Hong Kong-Macao Greater Bay Area (Table 1) show that the degree of correlation is larger in general, the sequence could be expressed as Construction $>$ Wholesale, Retail and Hotel catering $>$ Financial, Real estate and

Table 1. Synthetic correlation degree between tourism and related industries. 
other services $>$ Transportation and Telecommunications. The results demonstrated that construction industry has the closest correlation degree as high as 0.942 , compared with the relatively low correlation degree of Transportation, Post and Telecommunications, which turn out to be 0.555 . It reflects that the development of the tourism and leisure industry in Guangdong-Hong Kong-Macao Greater Bay Area has strong impetus to other related industries.

There are diversities of driving effects among different cities within the Guangdong-Hong Kong-Macao Greater Bay Area. From the perspective of construction industry, the sequence could be expressed as Guangzhou > Foshan > Zhongshan $>$ Zhuhai $>$ Macao $>$ Dongguan $>$ Zhaoqing $>$ Huizhou $>$ Jiangmen $>$ Shenzhen > Hong Kong; As for Wholesale, Retail and Hotel catering industry, the sequence could be expressed as Huizhou > Guangzhou > Dongguan $>$ Zhaoqing $>$ Foshan $>$ Macao $>$ Zhongshan $>$ Zhuhai $>$ Jiangmen $>$ Zhaoqing $>$ Zhuhai $>$ Hong Kong; Concerning Financial, Real estate and other services industry, the sequence could be expressed as Dongguan $>$ Zhaoqing $>$ Macao $>$ Zhuhai $>$ Jiangmen $>$ Guangzhou $>$ Huizhou $>$ Hong Kong $>$ Foshan $>$ Zhongshan > Shenzhen; in the matter of Transportation and Telecommunications industry, the sequence could be expressed as Shenzhen $>$ Dongguan $>$ Huizhou $>$ Macao $>$ Zhongshan $>$ Foshan $>$ Guangzhou $>$ Jiangmen $>$ Zhaoqing $>$ Zhuhai $>$ Hong Kong.

\subsection{Analysis of VAR Model}

\subsubsection{Model Specification}

In order to analyze the influence of the development of tourism on overall economy and investment in fixed assets within the Guangdong-Hong Kong-Macao Greater Bay Area. This section uses data from 2005 to 2014 establish a vector auto regressive (VAR) model of tourism revenue growth (RTRA), regional GDP growth (RGDP) and fixed asset investment growth (RK):

$$
\begin{aligned}
& \mathrm{RTRA}_{t}=\beta_{10}+\sum_{i=1}^{p} \beta_{1 i} \operatorname{RTRA}_{t-i}+\sum_{i=1}^{p} \alpha_{1 i} R G D P_{t-i}+\sum_{i=1}^{p} \gamma_{1 i} R K_{t-i}+\varepsilon_{1 i} \\
& \mathrm{RGDP}_{t}=\beta_{20}+\sum_{i=1}^{p} \beta_{2 i} R T R A_{t-i}+\sum_{i=1}^{p} \alpha_{2 i} R G D P_{t-i}+\sum_{i=1}^{p} \gamma_{2 i} R K_{t-i}+\varepsilon_{2 i} \\
& \mathrm{RK}_{t}=\beta_{30}+\sum_{i=1}^{p} \beta_{3 i} R T R A_{t-i}+\sum_{i=1}^{p} \alpha_{3 i} R G D P_{t-i}+\sum_{i=1}^{p} \gamma_{3 i} R K_{t-i}+\varepsilon_{3 i}
\end{aligned}
$$

\subsubsection{Lag Order Determination and Stationarity Test}

First of all, the lag order of the VAR model needs to be determined. Data analysis was performed with STATA software, the maximum lag order turned out to be two (Table 2). Then examines the stability of the model which lagged two periods, the results show that all the eigenvalues lie inside the unit circle, VAR satisfies stability condition (Figure 2), so the lag periods determined to be two.

\subsubsection{Analysis of Results}

First, the T-statistic for all lagged regression data is significant at level of $1 \%$ and the overall regression fit well (Table 3 ). The first-order lagged coefficients of RTRA on RGDP and RK are 0.208 and 0.799 , respectively. However, it is 
Table 2. Lag order determination of VAR model.

\begin{tabular}{ccccccccc}
\hline Lag & LL & LR & df & p & FPE & AIC & HQIC & SBIC \\
\hline 0 & 41.4131 & & & & $3.5 \mathrm{e}^{-09}$ & -10.9752 & -11.2617 & -10.9984 \\
1 & 67.2464 & 51.666 & 9 & 0.000 & $4.5 \mathrm{e}^{-11^{*}}$ & -15.7847 & -16.9307 & -15.8774 \\
2 & 755.442 & $1376.4^{*}$ & 9 & 0.000 & & $-209.841^{\star}$ & $-211.846^{*}$ & $-210.003^{*}$ \\
\hline
\end{tabular}

Table 3. Regression results.

\begin{tabular}{cccc}
\hline $\begin{array}{c}\text { Dependent } \\
\text { variable }\end{array}$ & RTRA & RGDP & RK \\
\hline Constant & $0.870^{* * *}$ & $0.0117^{* * *}$ & $0.356^{* * *}$ \\
& $(7.28 \mathrm{e}+14)$ & $(6.81 \mathrm{e}+13)$ & $(1.08 \mathrm{e}+15)$ \\
RTRA $(-1)$ & $1.809^{* * *}$ & $0.208^{* * *}$ & $0.799^{* * *}$ \\
& $(5.95 \mathrm{e}+14)$ & $(4.73 \mathrm{e}+14)$ & $(9.56 \mathrm{e}+14)$ \\
$\operatorname{RTRA}(-2)$ & $2.187^{* * *}$ & $-0.257^{* * *}$ & $0.809^{* * *}$ \\
& $(6.04 \mathrm{e}+14)$ & $(-4.92 \mathrm{e}+14)$ & $(8.13 \mathrm{e}+14)$ \\
$\operatorname{RGDP}(-1)$ & $-1.696^{* * *}$ & $0.0403^{* * *}$ & $-1.426^{* * *}$ \\
& $(-2.62 \mathrm{e}+14)$ & $(4.31 \mathrm{e}+13)$ & $(-8.01 \mathrm{e}+14)$ \\
$\operatorname{RGDP}(-2)$ & $-3.323^{* * *}$ & $0.191^{* * *}$ & $-1.104^{* * *}$ \\
& $(-5.04 \mathrm{e}+14)$ & $(2.01 \mathrm{e}+14)$ & $(-6.09 \mathrm{e}+14)$ \\
$\operatorname{RK}(-1)$ & $-5.679^{* * *}$ & $0.772^{* * *}$ & $-2.172^{* * *}$ \\
& $(-5.44 \mathrm{e}+14)$ & $(5.12 \mathrm{e}+14)$ & $(-7.58 \mathrm{e}+14)$ \\
$\operatorname{RK}(-2)$ & $-2.968^{* * *}$ & $-0.138^{* * *}$ & $-0.491^{* * *}$ \\
& $(-5.13 \mathrm{e}+14)$ & $(-1.65 \mathrm{e}+14)$ & $(-3.09 \mathrm{e}+14)$ \\
\hline
\end{tabular}

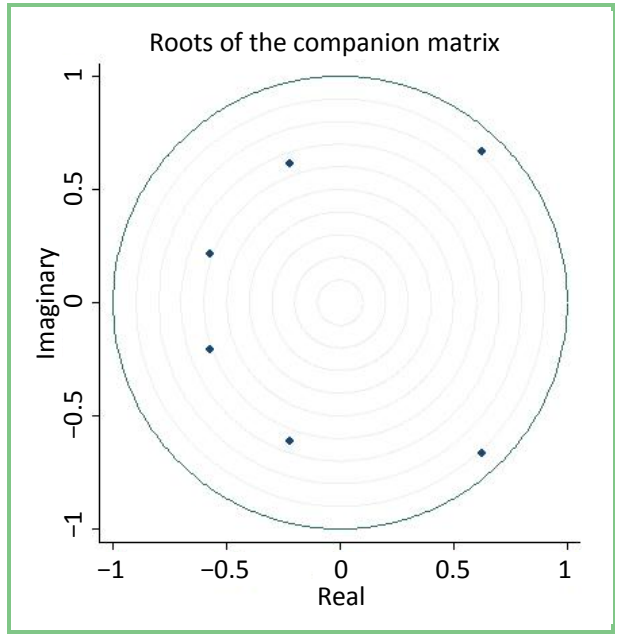

Figure 2. Stationarity test of VAR model.

noteworthy that the second-order coefficient of RTRA on RGDP is -0.257 , indicating that the tourism industry has a strong positive impact on investment in fixed assets, as for its driving effect on GDP, only worked in the short term.

\subsubsection{Granger Causality Test}

The Granger causality test was conducted on total tourism revenue (TRA), gross domestic product (GDP) and investment in fixed assets (K) (Table 4). In the 
Table 4. Result of Granger causality test.

\begin{tabular}{cccc}
\hline Equation & Excluded & chi2 & Prob $>$ chi2 \\
\hline TRA & GDP & 8.0091 & 0.018 \\
TRA & K & 11.816 & 0.003 \\
TRA & All & 25.71 & 0.000 \\
GDP & TRA & $4.5 \mathrm{e}+05$ & 0.000 \\
GDP & K & $6.3 \mathrm{e}+05$ & 0.000 \\
GDP & All & $2.7 \mathrm{e}+06$ & 0.000 \\
K & TRA & 8.9127 & 0.012 \\
K & GDP & 19.008 & 0.000 \\
K & All & 32.856 & 0.000 \\
\hline
\end{tabular}

equation which take the total income of tourism as a variable and take GDP as the inspection variables, the chi-square statistic of the GDP is 8.0091, and the corresponding P-value is 0.018 . Therefore, GDP can be regarded as Granger reason for total tourism revenue. Similarly, the amount of investment in fixed assets is also the Granger reason for total tourism revenue. At the same time, the joint significance of GDP and fixed asset investment was tested. The chi-square statistic was 25.71 and the corresponding P-value was approximately 0 . It strongly rejected the null hypothesis that "neither of these two explanatory variables is Granger's reason for total tourism revenue". In other words, both GDP and investment in fixed assets are the Granger reasons for total tourism revenue. The data in Table 4 also report the results which use GDP and investment in fixed assets as the explained variables, respectively. The results show that the total tourism revenue and investment in fixed assets are Granger causes of GDP, while the total tourism revenue and GDP are also Granger causes of total fixed assets investment. To sum up, the three variables exist two-way causality relationship rather than one-way causality relationship, indicating that there is an interdependence between the tourism and leisure industry, GDP and investment in fixed assets within Guangdong-Hong Kong-Macao Greater Bay Area.

\section{Conclusions and Recommendations}

Based on grey correlation analysis and vector auto regressive (VAR) model, this paper analyzed the driving effect of the tourism and leisure industry in Guangdong-Hong Kong-Macao Greater Bay Area. The results showed that the degree of correlation is larger in general, and the sequence could be expressed as Construction $>$ Wholesale, Retail and Hotel catering > Financial, Real estate and other services $>$ Transportation and Telecommunications. In addition, construction industry has the closest correlation degree as high as 0.942 , compared with the relatively low correlation degree of Transportation, Post and Telecommunications, which turns out to be 0.555 . It reflects that the development of the tourism and leisure industry in Guangdong-Hong Kong-Macao Greater Bay Area 
has strong impetus to other related industries. The results also demonstrated that the tourism industry has a strong positive impact on investment in fixed assets, but for its driving effect on GDP, only worked in the short term. It is noteworthy that those three variables exist two-way causality relationship rather than one-way causality relationship, indicating that there is an interdependence between the tourism and leisure industry, GDP and investment in fixed assets within Guangdong-Hong Kong-Macao Greater Bay Area. Based on results of the above research, several suggestions are put forward to promote the development of tourism and leisure industry in Guangdong-Hong Kong-Macao Greater Bay Area:

- Speed up the free exercise of yachts, accelerate investment in building public Wharf, popularize yacht tourism, and facilitate the development of leisure tourism and yacht manufacturing in Guangdong-Hong Kong-Macao Greater Bay Area. Under the flourishing situation of industry in Hong Kong and Macao is booming, more and more Yacht berth are needed. However, the yachts industry in the other city is still in the early stage, their Yacht berth would be remained idled. At present, only the free exercise of yachts between Zhongshan and Macao is established. If the scope of free exercise of yachts is broadened, other cities will be able to meet the needs of yacht mooring in Hong Kong and Macao and stimulate the development of yacht tourism in other cities. In addition, most of the marinas belong to the private clubs which are expensive, and the construction of public marina, which can provide low-cost berths are inadequate. If the government invests in the construction of public marina, it will bring about a reduction in the expenses of Yacht, thus promote the popularity and the development of the tourism and leisure industry in Guangdong-Hong Kong-Macao Greater Bay Area. Moreover, after the construction of public terminals, small and medium-sized yachts will be very popular and will be able to boost the development of manufacturing industry.

- Accelerating investment in the construction of the transport infrastructure enhances the rail transit and advances the construction of high expressways and promotes the close co-operation between urban tourism in Guangdong-Hong Kong-Macao Greater Bay Area. At present, the construction of rail transport within the Bay Area could not meet the demand satisfactorily, and the function of the airport port, train station and other large transport hub are lack of effective combination. Therefore, in order to promote the development of the tourism and leisure industry and the in-depth development of tourist routes in the region, it is necessary to deepen spatial planning and internal traffic organization, speed up the construction of expressways and airports, improve the accessibility and convenience of domestic and international tourists in Guangdong-Hong Kong-Macao Greater Bay Area.

- Increase the intensity of ecological protection and scientifically formulate the appropriate scale for the development of Guangdong-Hong Kong-Macao 
Greater Bay Area. Tourism development combines environmental protection and sabotage. With the increasing intensity of tourism development, urban and population are growing rapidly, but also lead to increased pressure on the ecological environment. The purpose of most tourists is recreation, and the most important factor that attracts the tourists is the beautiful coastal environment in the Bay Area. Therefore, in order to achieve a fast and sound sustainable development, it is necessary to control the scale and intensity of the ecological environment, scientifically formulate a suitable scale for the development of the coastal areas and increase the protection of the ecological environment. In addition, taking the natural geographical features of the Bay Area into account, the land resources that can be developed are relatively limited. Therefore, it is necessary to change the mode of land utilization moderately, and make rational planning and layout of the land resources of Guangdong-Hong Kong-Macao Greater Bay Area.

\section{References}

[1] Liu, Y.X. (2014) Research and Implications of Bay Zone Economy Home and Abroad. Urban Insight, 3, 155-163.

[2] Li, J.F., LI, G.L. and Jiang, B. (2001) An Analysis of Enterprise Relationship and Enterprise Spread in the Tourist Industry-Taking Guangdong as Example. Tourism Tribune, 3, 19-25.

[3] Song, Z.W. (2007) A Research on Industrial Correlation of China's Tourism Industry: Based on the Input-Output Model. Tourism Science, 2, 7-12+78.

[4] Weng, G.M., Zhang, J.C. and Lu, C. (2012) Industry Association Effects of Tourism in China. Enterprise Economy, 31, 127-130.

[5] Wu, S.M. (2012) An Analysis of Enterprise Relationship and Enterprise Spread in the China's Tourist Industry. Industrial Economics Research, 1, 78-86.

[6] Wang, Q.Y. and Xu, L. (2013) The Convergence of the Beijing Tourism and Agriculture Industries: Based on the Perspective of Industrial Relevance. Tourism Tribune, 28, 102-110.

[7] Huang, X.H. and Zou, K.M. (2016) A Study of the Integration Development of Culture, Commerce and Tourism under the Background of "the Belt and Road" Strategy in Guangdong-Hong Kong-Macao Greater Bay Area. Journal of South China Normal University (Social Science Edition), 4, 106-110 + 192. 